\title{
Entanglement Theory and the Second Law of Thermodynamics
}

\author{
Fernando G.S.L. Brandão ${ }^{1,2}$ \& Martin B. Plenio ${ }^{1,2}$ \\ QOLS, Blackett Lab, Imperial College London, Prince Consort Road, London SW7 2BW, UK and \\ Institute for Mathematical Sciences, Imperial College London, 53 Exhibition Road, London SW7 2PG, UK
}

(Dated: October 13, 2008)

\begin{abstract}
Entanglement is central both to the foundations of quantum theory and, as a novel resource, to quantum information science. The theory of entanglement establishes basic laws, such as the non-increase of entanglement under local operations, that govern its manipulation and aims to draw from them formal analogies to the second law of thermodynamics. However, while in the second law the entropy uniquely determines whether a state is adiabatically accessible from another, the manipulation of entanglement under local operations exhibits a fundamental irreversibility which prevents the existence of such an order.

Here we show that a reversible theory of entanglement and a rigorous relationship with thermodynamics may be established when one considers all non-entangling transformations. The role of the entropy in the second law is taken by the asymptotic relative entropy of entanglement in the basic law of entanglement. We show the usefulness of this new approach to general resource theories and to quantum information theory.
\end{abstract}

Thermodynamics is arguably one of the most fundamental and generally applicable theories of Nature whose foundations have remained intact despite the emergence of quantum mechanics, relativity and other physical laws $\stackrel{1}{1}$. It was initially understood to describe the physics of large systems in equilibrium, determining their bulk properties by a very simple set of rules of universal character. This was reflected in the formulation of the defining axiom of thermodynamics, the second law, by Clausius, Kelvin and Planck in terms of quasi-static processes and heat exchange. However, the apparently universal applicability of the theory suggested a deeper mathematical and structural foundation. Indeed, there is a long history of examinations of the foundations underlying the second law. Of particular interest in the present context is the work of Giles ${ }^{2}$ and notably Lieb and Yngvason ${ }^{3}$ stating that there exists a complete order for equilibrium thermodynamical states that determines which state transformations are possible by means of an adiabatic process. From simple, abstract, axioms they were able to show that this order is uniquely determined by an entropy function $S$ : given two equilibrium states $A$ and $B, A$ can be converted by an adiabatic process into $B$ if, and only if, $S(A) \leq S(B)$. As pointed out by Lieb and Yngvason 4 , it is a strength of this abstract approach that it allows to uncover a thermodynamical structure in settings that may at first appear unrelated.

One such possible setting is the theory of entanglement $t^{5, \underline{6}}$ in quantum information science ${ }^{\underline{7}}$. Although the importance of entanglement for the foundations of quantum mechanics has been noticed early $\underline{8,9}$, it was only over the last decade or so that the resource character of quantum correlations was recognized. A paradigmatic scenario in this respect is the one in which two distant parties want to exchange quantum information, but are restricted to act locally on their quantum systems and communicate classically. It is known that quantum state teleportation 10 overcomes the limitations caused by the restriction to local operations through the use of quantum mechanical correlations, entanglement. Entanglement theory is then concerned with the systematic exploration of entanglement as a resource in a quantitative manner.

Possible connections between entanglement theory and thermodynamics were noted when it was found that for bipartite pure states a very similar situation to the second law holds in the asymptotic limit of an arbitrarily large number of identical copies of the state. Then, given two bipartite pure states $\left|\psi_{A B}\right\rangle$ and $\left|\phi_{A B}\right\rangle$, the former can be converted into the latter by local operations and classical communication (LOCC) if, and only if, $E\left(\left|\psi_{A B}\right\rangle\right) \geq E\left(\left|\phi_{A B}\right\rangle\right)$, where $E$ is the entropy of entanglement, given by the von Neumann entropy of the reduced density matrix ${ }^{11}$.

However, for mixed entangled states there are bound entangled states 12 that contain quantum correlations and thus require a non-zero rate of pure state entanglement for their creation by LOCC, but from which no pure state entanglement can be extracted at all12,13,14. As a consequence no unique measure of entanglement exists in the general case and no unambiguous and rigorous direct connection to the second law appears possible despite various interesting attempts $15,16,17,18,19$.

Concepts and main results - In this work we identify the correct counterpart in entanglement theory of an adiabatic process. This allows us to establish a theorem completely analogous to the Lieb and Yngvason formulation of the second law of thermodynamics ${ }^{4}$ for entanglement manipulation. Similar considerations may also be applied to resources theories in general, including theories quantifying the non-classicality or the non-Gaussian character of quantum states. In the following we explain the basic relevant concepts, technical tools and the main result together with conclusions that we may draw from it.

Entangled states - We begin by delineating mathematically the boundary between classically and quantum correlated states for systems that consist of two parties each 
holding an arbitrary number of particles. A quantum state of such a system is described by a density operator $\rho$ acting on $\mathcal{H}_{A} \otimes \mathcal{H}_{B}$, where $\mathcal{H}_{A / B}$ are finite dimensional Hilbert spaces. This quantum state contains only classical correlations, and is called separable $\stackrel{20}{ }$, if there exist local density operators $\rho_{j}^{A / B}$ acting on $\mathcal{H}_{A / B}$ and a probability distribution $\left\{p_{j}\right\}$ such that

$$
\rho=\sum_{j} p_{j} \rho_{j}^{A} \otimes \rho_{j}^{B}
$$

Operationally, the set of separable states $\mathcal{S}$ is formed by all states that may be created from a pure product state $|0\rangle\langle 0|\otimes| 0\rangle\langle 0|$, i.e. an uncorrelated state, by means of LOCC. If $\rho$ cannot be written as in eq. (1) we say it is entangled and its generation requires, in addition to LOCC, an exchange of quantum particles (quantum communication) or a supply of pre-existing pure entangled states that are consumed in the process. A central state in this context is the two qubit maximally entangled state,

$$
\phi^{+}:=\frac{1}{2} \sum_{i, j=1}^{2}|i, i\rangle\langle j, j|,
$$

which defines the unit of entanglement $\mathrm{f}^{5}$.

The observation that LOCC alone does not create entanglement has been taken further to formulate as the basic law of entanglement manipulation that entanglement cannot be increased by LOCC. A principle that is similar in spirit to the second law of thermodynamics.

Asymptotic entanglement conversion - Our results are concerned with the asymptotic limit of a large number of identical copies of quantum states. We consider this scenario as we seek to indentify a total order for entanglement manipulation. For a finite number of copies, it is known that no such an order can exist, a fact that resembles the necessity of the thermodynamical limit in the context of the second law.

Here it is also natural to consider transformations between states that may be approximate for any finite number of systems and are only required to become exact in

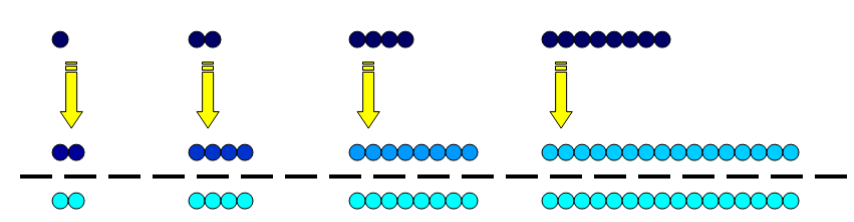

FIG. 1: Asymptotically entanglement conversion: The dark blue circles in the top row represent the initial state while the light blue coloured particles in the bottom row are the target state. The particles in the second row are the result of a quantum operation and approximate the third row increasingly well with increasing number of particles as indicated by the colour. Asymptotically the approximation becomes perfect. the asymptotic limit (see fig. 1). To describe this limiting process rigorously the well established trace distance $D(\rho, \sigma)=\|\rho-\sigma\|_{1}$ between quantum states is used. For two states that are close in trace distance the expectation value of any bounded physical observable will also be close when measured on the two states. Thus the trace distance quantifies how similar two states behave in physical experiments and is thus a sensible measure of distance between quantum states.

We say that a state $\rho$ can be asymptotically converted into another state $\sigma$ by operations of a given class if there is a sequence of quantum operations $\left\{\Psi_{n}\right\}$ within such a class acting on $n$ copies of the first state such that $\lim _{n \rightarrow \infty} D\left(\Psi_{n}\left(\rho^{\otimes n}\right), \sigma^{\otimes n}\right)=0$.

Measures of Entanglement - To formulate the main result we need two measures that will be used to quantify entanglement. It will be satisfying that these two seemingly distinct approaches will turn out to be equivalent in the asymptotic limit, as a consequence of the technical proof of our main theorem. Firstly, we consider the relative entropy of entanglement $\underline{18,24}$

$$
E_{R}(\rho)=\min _{\sigma \in \mathcal{S}} S(\rho \| \sigma),
$$

where $S(\rho \| \sigma)=\operatorname{tr}\{\rho \log \rho-\rho \log \sigma\}$ is the quantum relative entropy. and $\mathcal{S}$ is the set of separable states. Furthermore, we consider the global robustness

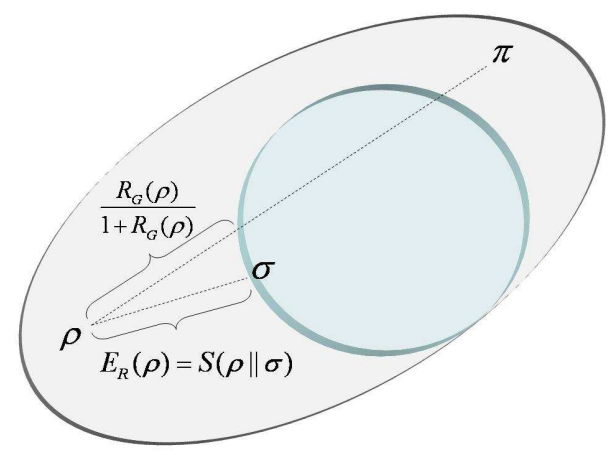

FIG. 2: Relative entropy of entanglement and global robustness of entanglement. Given an entangled state $\rho$, one can ask how distinguishable it is from a separable state $\sigma$. A good figure of merit is the quantum relative entropy $S(\rho \| \sigma)$ (see fig. 4). Following the intuition that the more entangled the more distinguishable a state is from a classically correlated one, we can then quantify the entanglement of $\rho$ by the minimum of $S(\rho \| \sigma)$ over all separable states $\sigma$, finding the measure known as relative entropy of entanglement $E_{R} 24$. Another approach to the quantification of entanglement is to look at the robustness of the quantum correlations contained in a state to global noise, from a geometrical point of view. Given an entangled state $\rho$, the global robustness of entanglement $R_{G}$ is the minimal amount of mixing $s$ of $\rho$ with another arbitrary state $\pi$ in order to turn the mixture $\frac{1}{1+s}(\rho+s \pi)$ into a separable state $e^{25,26}$. 
of entanglement $\frac{25,26}{2}$ wich is defined as

$$
R_{G}(\rho)=\min _{s \in \mathbb{R}}\left(s: \exists \sigma \text { s.t. } \frac{\rho+s \sigma}{1+s} \in \mathcal{S}\right) .
$$

Both are meaningful entanglement quantifiers whose physical motivations are outlined in figure 2. They will play a crucial role in the proof of our main theorem.

As we are concerned with the entanglement properties in the asymptotic limit we should not consider entanglement measures at the single copy level, but rather their asymptotic, or regularized, counterpart. In the case of $E_{R}$, the relevant quantity to consider is actually the regularized relative entropy of entanglement, given by

$$
E_{R}^{\infty}(\rho)=\lim _{n \rightarrow \infty} \frac{E_{R}\left(\rho^{\otimes n}\right)}{n} .
$$

This will turn out to be the central quantity in this work as it will emerge as the unique entanglement quantifier.

Operations - The correct choice of the set of operations employed is crucial for establishing reversibility in entanglement manipulation. To motivate this choice it is instructive to note that in the context of the second law it follows both from the approach of Giles ${ }^{2}$ as well as Lieb and Yngvason ${ }^{\underline{3}}$ that the class formed by all adiabatic processes is the largest class of operations which cannot decrease the entropy of an isolated equilibrium thermodynamical system.

Following such an inside we now identify the largest set of quantum operations that obeys the basic law of the non-increase of entanglement. Then one might expect to achieve reversibility in entanglement manipulation and thus a full analogy to the second law of thermodynamics. While operationally well motivated, the set of LOCC operations is not such a class.

The following choice of quantum operations represents a key insight that is crucial for establishing reversibility in entanglement theory. As we are concerned with asymptotic entanglement manipulation, it is physically natural and also convenient for mathematical reasons to define the set of asymptotically non-entangling operations, composed of sequences of operations that for a finite number of copies $n$ may generate a small amount $\epsilon_{n}$ of entanglement which vanishes asymptotically, $\lim _{n \rightarrow \infty} \epsilon_{n}=0$. It is important to note that we do not simply require that the entanglement per copy vanishes but actually the total amount of entanglement. More precisely, we call a quantum operation $\Omega$ a $\epsilon$-non-entangling operation if $R_{G}(\Omega(\sigma)) \leq \epsilon$ for every separable state $\sigma$. Thus the entanglement that is generated by the map is not robust to a small perturbation by $\epsilon$, which in particular implies that the state is $\epsilon$-indistinguishable from a separable state in trace norm. We then call a sequence of quantum operations $\left\{\Psi_{n}\right\}$ asymptotically non-entangling when each $\Psi_{n}$ is an $\epsilon_{n}$-non-entangling operation and $\lim _{n \rightarrow \infty} \epsilon_{n}=0$. This class is the largest class of quantum operations which cannot create entanglement in the limit of asymptotically many copies. Moreover, for the two measures

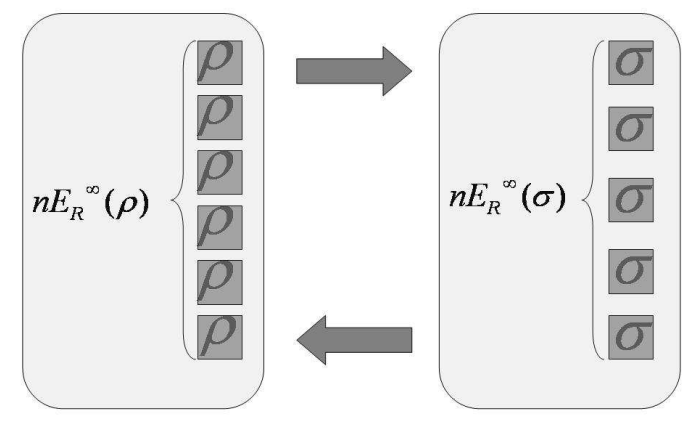

FIG. 3: Reversibility of entanglement manipulation. Entanglement under asymptotically non-entangling operations is a fungible resource: any two entangled states can be reversibly interconverted in the asymptotic limit $(n \rightarrow \infty)$, as long as the ratio of copies of each of them matches the ratio of the respective regularized relative entropies of entanglement.

we consider in this work, namely the relative entropy of entanglement and the global robustness of entanglement, it is also the largest class that asymptotically satisfies the law of the non-increase of entanglement.

To demonstrate reversibility of entanglement manipulation under the class just introduced we define two quantities and prove that they are the same. The entanglement cost under asymptotically nonentangling maps $E_{C}(\rho)$ is defined as the infimum of $\lim _{n \rightarrow \infty} \frac{k_{n}}{n}$ over all the sequences of quantum maps $\left\{\Psi_{n}\right\}$ and integers $\left\{k_{n}\right\}$, such that (i) each $\Psi_{n}$ is a $\epsilon_{n}$-non-entangling map, (ii) $\lim _{n \rightarrow \infty} \epsilon_{n}=0$, and (iii) $\lim _{n \rightarrow \infty} D\left(\Psi_{n}\left(\left(\phi^{+}\right)^{\otimes k_{n}}\right), \rho^{\otimes n}\right)=0$. Note that the map might add or take out particles from the system, so its input and output dimensions might differ.

Conversely, the distillable entanglement under asymptotically non-entangling maps $E_{D}(\rho)$ is given by the supremum of $\lim _{n \rightarrow \infty} \frac{k_{n}}{n}$ over all the sequences $\left\{\Psi_{n}\right\}$ and $\left\{k_{n}\right\}$ of quantum maps and integers satisfying conditions (i) and (ii) as in the paragraph above, but with the third requirement replaced by $\lim _{n \rightarrow \infty} D\left(\Psi_{n}\left(\rho^{\otimes n}\right),\left(\phi^{+}\right)^{\otimes k_{n}}\right)=0$.

The main theorem - Identifying the class of asymptotically non-entangling operations is of course not sufficient for our goals as it is not self-evident that this class will be sufficiently powerful to permit the existence of reversible interconversion of entangled resources. It is the main result of our work formulated in theorem I below to demonstrate that actually such a class does indeed lead to reversible transformations.

Theorem I - For every bipartite state $\rho$,

$$
E_{C}(\rho)=E_{D}(\rho)=E_{R}^{\infty}(\rho) .
$$

As shown in figure 3 , in this framework entanglement can be interconverted reversibly. The situation is formally analogous to the Lieb and Yngvason formulation 
of the second law of thermodynamics.

We have also identified $E_{R}^{\infty}$ as the natural counterpart to the entropy function in the second law. The relative entropy measures the distinguishability of two quantum states (see figures 2 and 4), an observation that plays an important role in the technical proof. Therefore, in our framework, the amount of entanglement of a given state is completely determined by how distinguishable it is from a state that only contains classical correlations.

An outline of the proof of theorem I is given in the Methods section. We use techniques from quantum hypothesis testing 21,23 (see figure 4), which we generalize to tackle also non-identical-and-idependently-distributed instances, together with ideas from convex optimization and duality, applied to the cone of separable states, recent results on the characterization of symmetric states 22 , and several properties of entanglement measures that have been established in recent years 5.6 .

Implications and Applications - Theorem I has further consequences and implications that we would like to explore in the remainder of this work.

Entanglement Theory: Firstly, it sheds some light on the origin of the irreversibility in the LOCC setting and on the associated phenomenon of bound entanglement 12 . Indeed, our result suggests that such an irreversibility could have its roots on the assymmetry of operations that can be locally implemented without any entanglement (LOCC) and the operations that do not generate any entanglement. Moreover, it also represents a conterargument to linking irreversibility to the loss of classical information when going from pure to mixed states.

Secondly, the uniqueness theorem we established for mixed-state entanglement measures is an important new conceptual tool in entanglement theory. Indeed, we have seen already that the insight of considering entanglement manipulation under asymptotic non-entangling maps have allowed for the identification of the global robustness and the relative entropy of entanglement in the asymptotic regime, two important measures of entanglement that were believed to be unrelated. In fact, theorem I implies that any measure which is (i) asymptotically continuous ${ }^{5,6}$, (ii) monotonic under $\epsilon$ non-entangling maps in the limit $\epsilon \rightarrow 0$, and (iii) normalized, having a value of $n$ for $\phi_{+}^{\otimes n}$, regularizes to $E_{R}^{\infty}$. From this result it is possible to identify new measures that are useful both as sharp bounds for the LOCC distillable entanglement and also as a first step towards the derivation of a single-letter expression for the regularized relative entropy of entanglement, i.e. a formula without needing the limit of a infinite number of copies of the state as in eq. (5).

Even more complicated than bipartite mixed state entanglement manipulation is the case of of multipartite entanglement, where already for pure states little is known about entanglement conversion under LOCC ${ }^{5,6}$. Our approach can be readily generalized to the multipartite setting, leading again to an unique measure of entanglement, the regularized relative entropy of entanglement with re- spect to the set of fully separable states, completely specifying states transformations under asymptotically nonentangling operations.

General resource theories: The approach of considering the manipulation of a given resource under the largest class of operations that cannot create it is appealing and can also be applied to other resource theories.

One example is the study of the non-classicality of quantum states of continuous variable systems describing e.g. modes of the electromagnetic field, nano-electromechanical oscillators or quantum fluctuations of large atomic ensembles. Coherent states and convex combinations thereof are usually regarded as classical, whereas any other state is said to be non-classical. We can define the set of classical operations as the set of operations that maps every classical state to another classical state. Non-classical states can then be seen as a resource to implement general operations using only classical ones. The study of the manipulation of non-classical states by such operations emerges as a natural analog to entanglement theory and indeed basic features such as the non-increase of the degree of non-classicality under classical operations can be recovered. Both the relative entropy of entanglement and the robustness of entanglement have direct counterparts for the quantification of non-classicality, obtained by replacing in their definition the set of separable states set with the set of classical states. Hence we can also define the class of asymptotically classical maps as we did before for entanglement.

A paradigmatic example of a non-classical state is the so-called cat state, given by $\left|\phi_{\alpha}\right\rangle:=(2(1-$ $\left.\left.e^{-|2 \alpha|^{2}}\right)\right)^{-1 / 2}(|\alpha\rangle-|-\alpha\rangle)$, where $|\alpha\rangle$ is a coherent state with mean number of photons $|\alpha|^{2}$. We define in analogy to entanglement theory the cost of forming, with classical operations, a state out of many copies of $\left|\phi_{\alpha}\right\rangle$ and the distillation rate of obtaining it from many copies of a given non-classical state. Interestingly, we can employ the same techniques of the proof of theorem I to show that the cost function in the case where $|\alpha| \rightarrow \infty$ converges to the asymptotic global robustness of nonclassicality, defined in analogy to eq. (7). This is result is remarkable because, in contrast to entanglement theory, it is not a priori clear that we could define a unit of non-classicality from which any other non-classical state could be formed. It is possible not only to identify the cat state of a very large number of excitations as such pure form of non-classicality but also provide a closed form formula for the cost under asymptotically classical maps.

Another example of such a resource theory concerns the Gaussian character of quantum states. This is of relevance in continuous variable implementations of quantum information processing in the optical regime, where Gaussian states are most easily accessible ${ }^{32}$. Results analogous to the above can be obtained for the nonGaussianity of quantum states as well, where now any quantum state that cannot be written as a convex combination of states with a Gaussian Wigner function is 
treated as a resource.

The paradigm that we put forward could also potentially be helpful in the sudy of non-locality of quantum states, of super-selections rules 30 and even beyond the real of quantum physics, in the study of secret correlations of tripartite probability distributions, in cryptography 31 .

Entanglement and Thermodynamics: To conclude we would like to point out an intriguing possibility raised by our findings. Whereas Giles ${ }^{2}$ as well as Lieb and Yngvason ${ }^{3,4}$ derived the total order for thermodynamical states from a set of postulates that intuitively should hold for thermodynamics, we have proved the existence of a total order for entangled states from the axioms of quantum theory. From theorem I we can do the opposite and try to derive the axioms proposed by Lieb and Yngvason ${ }^{3,4}$ as consequences of the total order. Indeed all but one can be derived, but currently we cannot rule out the existence of catalysis 33 in the resource theory we devised for entanglement: there could be triplet of states $\rho, \pi$ and $\sigma$ such that the transformation $\rho \rightarrow \pi$ is not possible, but become feasible under the presence of the catalyst $\sigma$, i.e. $\rho \otimes \sigma \rightarrow \pi \otimes \sigma$. The non-existence of catalysis, however, is one of the axioms posed by Lieb and Yngvason. Interestingly, it can be readily seen that the existence of catalysts is equivalent to the non additivity of the entropy function in our theory, given by $E_{R}^{\infty}$, which is an open problem in entanglement theory, with implication also to the manipulation of entanglement under local operations and classical communication ${ }^{34}$.

The results of this paper show that entanglement theory, and in fact general resource theories satifying certain properties, have a thermodynamical flavor in its structural form. Indeed, such a link might be seen as an indication of the success of Giles ${ }^{2}$ and Lieb and Yngvason ${ }^{3}$ approach in identifying the underlying mathematical and logical structure behind the second law.

\section{METHODS}

We present an overview of the main steps taken in order to establish eq. (6). The argument is rather involved and it is therefore out of the scope of this paper to present all the details. A rigorous proof of theorem $\mathrm{I}$ is given in ref. 26. The proof can be divided in three parts.

In the first, we connect $E_{R}^{\infty}$ to the global robustness of entanglement. Let us define

$L G(\rho):=\inf _{\left\{\rho_{n} \in \mathcal{D}\left(\mathcal{H}^{\otimes n}\right): D\left(\rho_{n}, \rho^{\otimes n}\right) \rightarrow 0\right\}}\left\{\lim _{n \rightarrow \infty} \frac{L R_{G}\left(\rho_{n}\right)}{n}\right\}$,

where $L R_{G}(\rho):=\log \left(1+R_{G}(\rho)\right)$. This quantity, which we call asymptotic global robustness of entanglement, is a proper modification of the global robustness of entanglement so that it becomes a meaningful quantity in asymptotic considerations. A first step towards eq. (6) is the proof that for every state $\rho$,

$$
E_{R}^{\infty}(\rho)=L G(\rho) .
$$

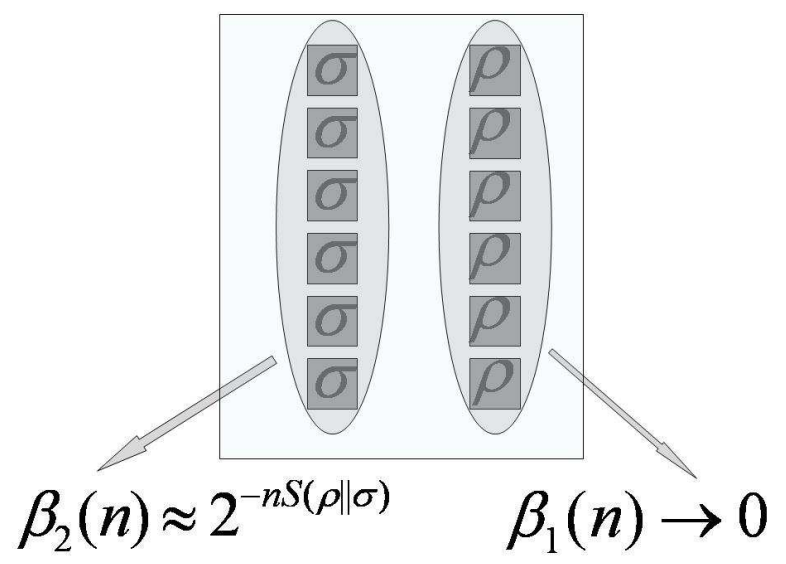

FIG. 4: Quantum Stein's Lemma. In quantum hypothesis testing an observer is given several identical copies of an unknown quantum state with the promise that it is described either by the density matrix $\rho$ or $\sigma$. His task is to perform measurements on these copies in order to find out which is the true state of the system. There are two types of errors in the process: $\beta_{1}(n)\left(\beta_{2}(n)\right)$ is the probability that, after performing a measurement on $n$ copies, the observer concludes that the system was described by the state $\sigma(\rho)$ when in reality it was described by $\rho(\sigma)$. Quantum Stein's Lemma gives the optimal rate of the exponential decay of $\beta_{2}(n)$ in the limit of infinitely many copies, when one requires that $\beta_{1}(n) \rightarrow 0$ asymptotically. Its direct part states that any number smaller than $S(\rho \| \sigma)$ is an achievable rate. This can be mathematically expressed in a compact manner as $\operatorname{tr}\left(\rho^{\otimes n}-2^{y n} \sigma^{\otimes n}\right)_{+} \rightarrow 1$ whenever $y<S(\rho \| \sigma)^{21}$. The converse part, in turn, establishes that there is no test with a better rate than the relative entropy, i.e. $\operatorname{tr}\left(\rho^{\otimes n}-2^{y n} \sigma^{\otimes n}\right)_{+} \rightarrow 0$ for every $y>S(\rho \| \sigma)^{23}$. Here $\operatorname{tr}(A)_{+}$stands for the sum of the positive eigenvalues of $A$.

The inequality $E_{R}^{\infty}(\rho) \leq L G(\rho)$ follows from the operator monotonicity of the log function together with the asymptotic continuity of $E_{R}{ }^{29}$. The converse inequality, in turn, can be derived from the converse part of quantum Stein's Lemma, explained in fig. 4. Essentially, if $y>S(\rho \| \sigma)$, then, for sufficiently large $n$, we have $\rho_{n} \leq 2^{y n} \sigma^{\otimes n}$, for an approximation $\rho_{n}$ of $\rho^{\otimes n}$ such that $D\left(\rho^{\otimes n}, \rho_{n}\right) \rightarrow 0$ asymptotically. If we take $\sigma$ as a separable state such that $E_{R}(\rho)=S(\rho \| \sigma)$, we find from the definition of $L R_{G}$ that $L R_{G}\left(\rho_{n}\right) / n \leq E_{R}(\rho)$, from which follows that $L G(\rho) \leq E_{R}(\rho)$. Finally, we use that $L G$ is weakly-additive to obtain eq. (8).

In the second part, we show that for every state $\rho$,

$$
E_{C}(\rho)=E_{R}^{\infty}(\rho)
$$

This is obtained by showing that $E_{C}(\rho)=L G(\rho)$, which together with eq. (8) implies eq. (9). That $E_{C}(\rho) \geq$ $L G(\rho)$ can be established from the fact that $L R_{G}$ can be increased by a $\epsilon$-non-entangling map by no more than $\log (1+\epsilon)$. To prove the converse inequality, we construct a sequence of $\epsilon_{n}$-non-entangling map achieving the lower 
bound $L G(\rho)$. We consider the sequence of maps

$$
\begin{aligned}
\Psi_{n}(A)= & \operatorname{tr}\left(A\left(\phi^{+}\right)^{\otimes k_{n}}\right) \rho_{n} \\
& +\operatorname{tr}\left(A\left(\mathbb{I}-\left(\phi^{+}\right)^{\otimes k_{n}}\right)\right) \pi_{n},
\end{aligned}
$$

where $\left\{\rho_{n}\right\}$ is an optimal sequence of approximations for $\rho^{\otimes n}$ in eq. (17) and $\pi_{n}$ an optimal state for $\rho_{n}$ in the global robustness of entanglement. By the convexity of $R_{G}{ }^{25,26}$ and the fact that the maximal overlap of $\left(\phi^{+}\right)^{\otimes k_{n}}$ with a separable state is $1 / 2^{k_{n}}$, we find that by choosing $k_{n}=L R_{G}\left(\rho_{n}\right)$, each $\Psi_{n}$ is a $R_{G}\left(\pi_{n}\right)$-nonentangling map. Then, we use that (i) $R_{G}\left(\rho_{n}\right) \rightarrow \infty$ and (ii) $R_{G}\left(\pi_{n}\right) \leq 1 / R_{G}\left(\rho_{n}\right)$ to see that $\epsilon_{n}=R_{G}\left(\pi_{n}\right) \rightarrow 0$ asymptotically. Fact (ii) follows directly from the definiion of $R_{G}$, while fact (i) is a consequence of the quantum de Finetti theorem ${ }^{22}$. We thus find $\left\{\Psi_{n}\right\}$ as an admissible sequence of maps for $E_{C}$, from which follows that indeed $E_{C}(\rho) \geq L G(\rho)$.

In the third and last part we show that $E_{D}(\rho)=$ $E_{R}^{\infty}(\rho)$, completing the proof. That $E_{D}(\rho) \leq E_{R}^{\infty}(\rho)$ can be established from the asymptotic continuity of the relative entropy of entanglement ${ }^{29}$ and the fact that also $E_{R}$ can be increased by a $\epsilon$-non-entangling map by no more than $\log (1+\epsilon)$.

To show the converse inequality, we proceed in two steps. The first is to derive a more handy expression for the singlet-fraction under non-entangling maps, defined as $F_{\text {sep }}\left(\rho, 2^{r}\right):=\max _{\Psi} \operatorname{tr}\left(\Psi(\rho)\left(\phi^{+}\right)^{\otimes r}\right)$, where the maximum is taken over all non-entangling operations $\Psi$. By using arguments from convex optimization and duality, it can be shown, in analogy with what was done for PPT maps in ref. 27 , that

$$
F_{\text {sep }}\left(\rho^{\otimes n} ; 2^{n D}\right)=\min _{\sigma \in \mathcal{S}, b \in \mathbb{R}} \operatorname{tr}\left(\rho^{\otimes n}-2^{r n} \sigma\right)_{+}+2^{(b-D) n},
$$

where $\mathcal{S}$ is the set of separable states. If $D$ is such that $\lim _{n \rightarrow \infty} F_{\text {sep }}\left(\rho^{\otimes n} ; D\right)=1$, then it is clear that $E_{D}(\rho) \geq$ $D$. From the direct part of quantum Stein's Lemma we have that $\operatorname{tr}\left(\rho^{\otimes n}-2^{y n} \sigma^{\otimes n}\right)_{+} \rightarrow 1$ whenever $y<S(\rho \| \sigma)$ (see fig. 4). The key point of the proof is that a very similar situation holds for $\min _{\sigma_{n} \in \mathcal{S}} \operatorname{tr}\left(\rho^{\otimes n}-2^{r n} \sigma_{n}\right)_{+}$, if we replace $S(\rho \| \sigma)$ by $E_{R}^{\infty}(\rho)$. Using the exponential de Finetti theorem recently proved by Renner ${ }^{22}$, we show that

$$
\lim _{n \rightarrow \infty} \min _{\sigma_{n} \in \mathcal{S}} \operatorname{tr}\left(\rho^{\otimes n}-2^{r n} \sigma_{n}\right)_{+}=1
$$

for $r<E_{R}^{\infty}(\rho)$, which combined with eq. (11) readily implies that $E_{D}(\rho) \geq E_{R}^{\infty}(\rho)$.

Correspondence - Correspondence and requests for materials should be addressed to Fernando Brandão. (Email: fernando.brandao@imperial.ac.uk)

Acknowledgements - We gratefully thank K. Audenaert, J. Eisert, A. Grudka, M. Horodecki, R. Horodecki, S. Virmani, and R.F. Werner for useful discussions and correspondence. This work is part of the QIP-IRC supproted by EPSRC and the Integrated Project Qubit Applications (QAP) supported by the IST diectorate and was supported by the Brazilian agency Conselho Nacional de Desenvolvimento Científico e Tecnológico (CNPq) and a Royal Society Society Wolfson Research Merit Award.

Competing Interests - The authors declare that they have no competing financial interests.
1 Callen, H.B. Thermodynamics and an Introduction to Thermostatistics, John Wiley and Sons (1985).

${ }^{2}$ Giles, R. Mathematical Foundantions of Thermodynamics, Pergamon, Oxford, 1964.

3 Lieb, E.H. and Yngvason, J. The Physics and Mathematics of the Second Law of Thermodynamics. Phys. Rept. 310, 1-96 (1999).

4 Lieb, E.H. and Yngvason, J. The Mathematical Structure of the Second Law of Thermodynamics. Current Developments in Mathematics, 2001 89, International Press (2002).

5 Plenio, M.B. and Virmani, S. An introduction to entanglement measures. Quant. Inf. Comp. 7, 1-51 (2007).

${ }^{6}$ Horodecki, R. Horodecki, P. Horodecki, M. and Horodecki, K. Quantum entanglement. quant-ph/0702225.

7 Bennett, C.H. and Divincenzo D.P. Quantum Information and Computation. Nature 404, 247-255 (2000).

8 Einstein, A. Podolsky, B. and Rosen, N. Can quantum mechanical description of physical reality be considered complete? Phys. Rev. 47, 777-780 (1935).

9 Schrödinger, E. Die gegenwärtige Situation in der Quan- tenmechanik. Naturwissenschaften 23, 807-812 (1935).

10 Bennett, C.H. Brassard, G. Crepeau, C. Jozsa, R. Peres, A. Wootters, W.K. Teleporting an unknown quantum state via dual classical and Einstein-Podolsky-Rosen channels. Phys. Rev. Lett. 70, 1895-1899 (1992).

11 Bennett, C.H. Bernstein, H.J. Popescu, S. and Schumacher, B. Concentrating partial entanglement by local operations. Phys. Rev. A 53, 2046-2052 (1996).

12 Horodecki, M. Horodecki, P. and Horodecki, R. MixedState Entanglement and Distillation: Is there a Bound Entanglement in Nature? Phys. Rev. Lett. 80, 5239-5242 (1998).

13 Vidal, G. and Cirac, J.I. Irreversibility in Asymptotic Manipulations of Entanglement. Phys. Rev. Lett. 86, 58035806 (2001).

14 Yang, D. Horodecki, M. Horodecki, R. and Synak-Radtke, B. Irreversibility for all bound entangled states. Phys. Rev. Lett. 95, 190501-190504 (2005).

15 Popescu, S. and Rohrlich, D. Thermodynamics and the Measure of Entanglement. Phys. Rev. A 56, R3319-R3321 (1997). 
16 Horodecki, P. Horodecki, R. and Horodecki, M. Entanglement and thermodynamical analogies. Acta Phys. Slov. 48, 141-156 (1998).

17 Plenio, M.B. and Vedral, V. Entanglement in Quantum Information Theory. Contemp. Phys. 39, 431-466 (1998).

18 Vedral, V. and Plenio, M.B. Entanglement Measures and Purification Procedures. Phys. Rev. A 57, 1619-1633 (1998).

19 Horodecki, M. Oppenheim, J. and Horodecki, R. Are the laws of entanglement theory thermodynamical? Phys. Rev. Lett. 89240403 (2002).

${ }^{20}$ Werner, R.F. Quantum states with Einstein-PodolskyRosen correlations admitting a hidden-variable model. Phys. Rev. A 40, 4277-4281 (1989).

21 Hiai, F. and Petz, D. The Proper Formula for Relative Entropy and its Asymptotics in Quantum Probability. Commun. Math. Phys. 143, 99-114 (1991).

22 Renner, R. Symmetry of large physical systems implies independence of subsystems. Nature Physics 3, 645-649 (2007).

23 Ogawa, T. and Nagaoka, H. Strong Converse and Stein's Lemma in the Quantum Hypothesis Testing. IEEE Trans. Inf. Theo. 46, 2428-2433 (2000).

24 Vedral, V. Plenio, M.B. Rippin, M.A. and Knight, P.L. Quantifying entanglement. Phys. Rev. Lett. 78, 2275-2279 (1997).

25 Vidal, G. and Tarrach, R. Robustness of entanglement. Phys. Rev. A 59, 141-155 (1999).
${ }^{26}$ Harrow, A.W. and Nielsen, M.A. How robust is a quantum gate in the presence of noise? Phys. Rev. A 68, 012308012321 (2003).

27 Brandão, F.G.S.L. and Plenio, M.B. Reversibility of entanglement manipulation under non-entangling operations. arXiv:0710.5827 [quant-ph].

28 Rains, E.M. A semidefinite program for distillable entanglement. IEEE T. Inform. Theory 47, 2921-2933 (2001).

29 Donald, M.J. and Horodecki, M. Continuity of Relative Entropy of Entanglement. Phys. Lett. A 264, 257-260 (1999).

30 Gour, G. and Spekkens, R.W. The resource theory of quantum reference frames. arXiv:07110043 [quant-ph].

31 Maurer, U. Secret key agreement by public discussion from common information. IEEE Trans. Inf. Theo. 39, 733-742 (1993).

32 Eisert, J. and Plenio, M.B. Introduction to the basics of entanglement theory in continuous-variable systems. Int. J. Quant. Inf. 1, 479-506 (2003).

33 Jonathan, D. and Plenio, M.B. Entanglement-assisted local manipulation of pure quantum states. Phys. Rev. Lett. 83, 3566-3569 (1999).

34 Brandão, F.G.S.L. Horodecki, M. Plenio, M.B. and Virmani, S. Remarks on the equivalence of full additivity and monotonicity for the entanglement cost. Open System 83 Information Dynamics 14, 333-339 (2007). 